\title{
Access to Medical Care for Individuals with Myalgic Encephalomyelitis and Chronic Fatigue Syndrome: A Call for Centers of Excellence
}

\author{
Madison Sunnquist ${ }^{1}$, Laura Nicholson ${ }^{1}$, Leonard A. Jason ${ }^{1 *}$, and Kenneth J. Friedman ${ }^{2}$ \\ ${ }^{1}$ Center for Community Research, DePaul University, Chicago, Illinois, United States \\ ${ }^{2}$ Green Mountain College, Poultney, Vermont, United States \\ Email: 1jason@depaul.edu
}

\begin{abstract}
The current study sought to better understand the experience of individuals with myalgic encephalomyelitis (ME) and chronic fatigue syndrome (CFS) in accessing care for their debilitating illness. Of 898 participants, less than half had ever seen an ME or CFS specialist, though $99 \%$ of participants were interested in specialist care. Participants cited geographic and financial barriers as most frequently precluding access to specialists. Furthermore, satisfaction with specialist care greatly exceeded satisfaction with non-specialist care. These findings suggested that individuals with ME and CFS represent a medically-underserved population, due to lack of available care. The CFS Advisory Committee and NIH Pathways to Prevention Working Group recommended the creation of ME and CFS Centers of Excellence to improve the healthcare access of patients with ME and CFS. The current study documents the need for these centers, as they would ameliorate geographic and financial barriers to quality care.
\end{abstract}

Keywords: Myalgic encephalomyelitis, chronic fatigue syndrome, healthcare access, specialist care, satisfaction with care, centers of excellence, medically-underserved population

\section{Introduction}

Myalgic Encephalomyelitis (ME) and Chronic Fatigue Syndrome (CFS) are characterized by a complex illness process that affects multiple systems within the body. Although the multi-system, disabling nature of ME and CFS calls for expert care, patients often report that their medical needs are unmet. A recent study [1] revealed that $54 \%$ of U.S. patients with ME or CFS reported dissatisfaction with the medical care that they received for their illness. Specifically, they attributed their dissatisfaction to physicians' inadequate training in treating individuals with their illness. Supporting this view, $71 \%$ of participants in this survey needed to see four or more physicians in order to receive a diagnosis.

Lack of curriculum exposure in medical school and clinical exposure in residency programs and teaching hospitals may contribute to issues of misdiagnosis and inadequate treatment of ME and CFS. A review of 119 medical textbooks found that fewer than half mentioned ME or CFS, and only $21 \%$ reviewed ME or CFS diagnostic criteria. [2] Inadequate representation of ME and CFS in medical education impacts the quality of care that patients receive. A study of physician attitudes and knowledge about ME and CFS revealed that approximately half of the surveyed physicians were unaware of diagnostic criteria, lacked confidence in diagnosing the illness, and felt uncomfortable treating patients. [3]

Lack of access to knowledgeable healthcare providers restricts the quality of care that individuals with ME and CFS can receive. The creation of Centers of Excellence has been recommended to address the issue of insufficient access to healthcare and patient dissatisfaction with medical care. [4,5] A CFS Advisory Committee (CFSAC) working group has argued that the creation of a network of investigators, clinicians, and educators would allow for new interventions, appropriate training models, and increased access to care for patients. [6] Online meeting notes indicate that CFSAC recommendations for Centers of Excellence date back to the year 2004. [7] To further explore the need for Centers of Excellence, the current study examined access to ME and CFS specialist care, barriers to accessing specialist care, and 
patients' satisfaction with specialist and general, non-specialist care.

\section{Method}

A survey was constructed to assess participants' access to ME and CFS specialist care, barriers to specialist care, and satisfaction with care received from both specialists and non-specialists. In order to enable severely ill individuals to participate, the survey was kept brief, solely targeting issues of healthcare access and satisfaction with care. The survey was hosted on Research Electronic Data Capture (REDCap), an online data collection tool. [8]

To be eligible, participants needed to have a self-reported diagnosis of ME or CFS, be 18 years of age or older, and be capable of reading and comprehending English. While this report was limited to the analysis of data obtained from U.S.-based participants, data were also collected from individuals residing outside of the United States; these data would be reported elsewhere. Approval for this study was obtained from DePaul University's Institutional Review Board prior to participant recruitment. Participants were recruited from announcements in ME and CFS organization newsletters and postings on social media accounts and online patient forums.

\section{Results}

Table 1 displays demographic information for the 898 U.S. participants. The majority of participants were female (90\%) and Caucasian (96\%). Approximately 56\% were married; $19 \%$ had never been married, and the remaining $25 \%$ were separated, widowed, or divorced. Regarding educational attainment, $31 \%$ held a graduate or professional degree; $36 \%$ had completed college; $31 \%$ were high school graduates; and $2 \%$ had not completed high school. Almost half of the sample (49\%) was on disability; $14 \%$ were unemployed; $13 \%$ had retired; $9 \%$ were working part-time; $8 \%$ were working fulltime; and the remainder $(8 \%)$ were students or homemakers.

Table 1. Demographics $(n=898)$

\begin{tabular}{lc}
\hline & $\mathrm{M}(S D)$ \\
\hline Age & $53.4(11.9)$ \\
Length of Illness & $18.3(11.1)$
\end{tabular}

\begin{tabular}{lc}
\hline & $\%(\mathrm{n})$ \\
\hline Gender & \\
Female & $89.5(797)$ \\
Male & $10.3(92)$ \\
Other & $0.2(2)$
\end{tabular}

Race (could select more than one option)

White

American Indian/Alaskan Native

Asian/Pacific Islander

African American

$1.6(14)$

Other

Latino or Hispanic Origin

No

Yes

Education Level

Graduate / Professional degree 
High school degree

Some high school

Less than high school

Work Status

On disability

Unemployed

$13.9(125)$

Retired

Working part-time

Working full-time

$7.6(68)$

Homemaker

Student

Marital Status

Married / Living with partner

Divorced

Never married

$19.6(176)$

Separated

Widowed

U.S. Region

Pacific

South Atlantic

$18.2(163)$

East North Central

$14.3(128)$

Middle Atlantic

Mountain

$9.7(87)$

$7.2(65)$

New England

$5.9(53)$

West South Central

West North Central $3.3(30)$

Over half $(52.2 \%)$ of participants reported that they had never seen a specialist; only $11.6 \%$ reported that a specialist was their regular care provider. Most participants who had seen a specialist had only seen the specialist for a test or referral in the past $(22.6 \%) ; 11.2 \%$ reported having seen a specialist on a consultative basis, but continued to see a non-specialist physician for their regular care. The remainder $(2.3 \%)$ had seen a specialist in the past, but did not report how frequently they saw the specialist.

Table 2. Barriers to specialist access $(n=469)$

\begin{tabular}{lc}
\hline Barrier & \% Endorsement \\
\hline No specialists in geographic area & 71.9 \\
Appointment costs too much & 32.0 \\
Health insurance does not cover appointment with specialist & 24.3 \\
Not healthy enough to travel to specialist's office & 23.2 \\
Traveling to appointment costs too much & 20.5 \\
Specialist does not accept Medicaid / Medicare & 15.8 \\
No transportation to specialist's office & 6.8 \\
No health insurance & 5.3 \\
Symptoms prevented from attending scheduled appointment & 3.8 \\
Responsibilities precluded scheduling an appointment & 2.6 \\
Inconvenient office hours & 1.5 \\
Not interested & 1.1 \\
\hline
\end{tabular}

Participants who had never seen a specialist were asked about potential barriers to accessing specialist 
care. Table 2 displays the percentage of these participants who endorsed each barrier listed in the survey. A large majority of participants indicated that geographic barriers prevented them from accessing specialist care: $72 \%$ of participants reported that there were no specialists in their area; $23 \%$ stated that they were not healthy enough to travel to a specialist's office; and $20 \%$ reported that traveling to a specialist's office would cost too much. Financial barriers were also a deterrent to specialist care: $32 \%$ reported that the specialist appointment would costs too much, and $24 \%$ of participants did not have insurance that would cover specialist appointments. Only $1 \%$ of participants indicated that they were not interested in seeing a specialist.

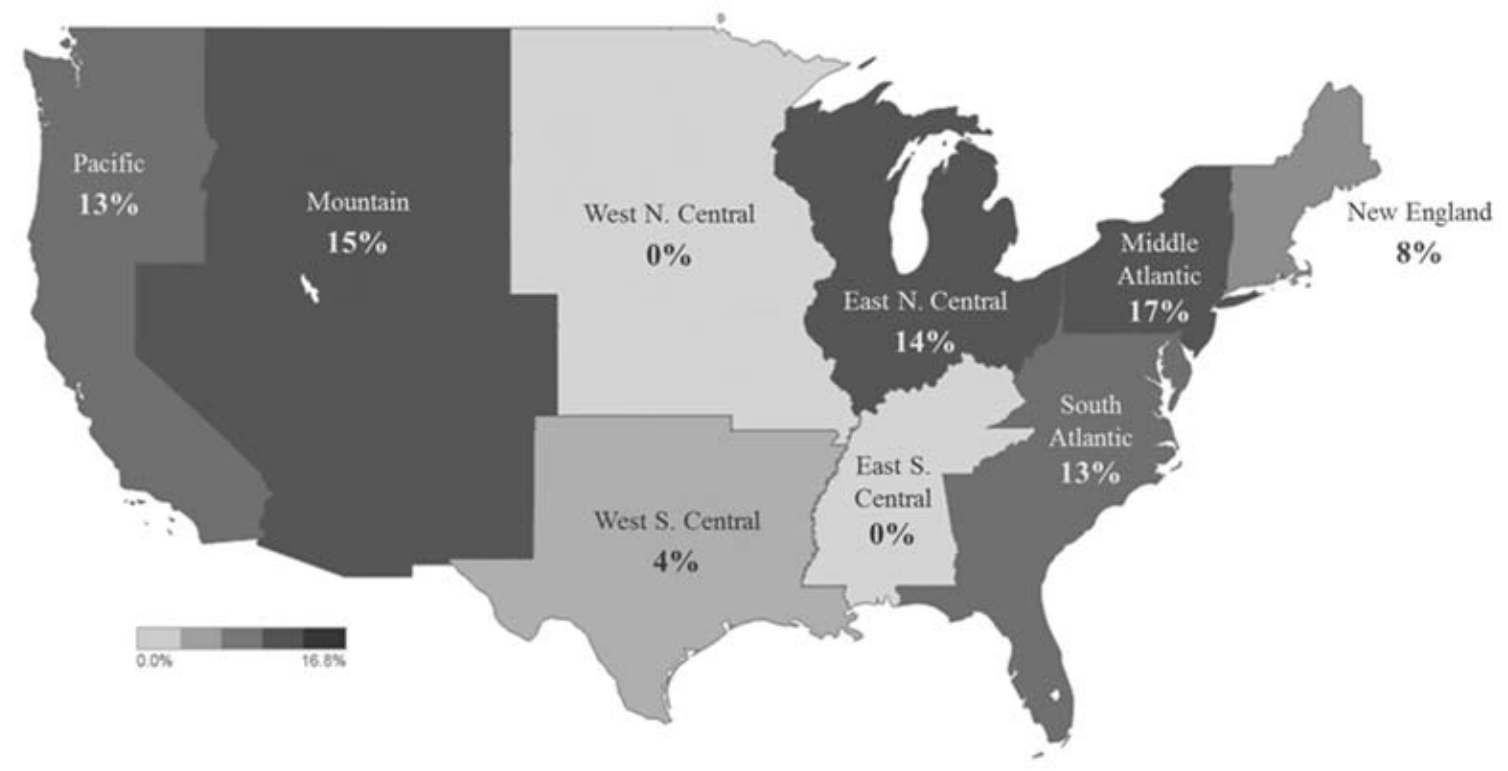

Figure 1. Percentage of participants who see a specialist as their primary ME or CFS care provider by U.S. Census Region.

Findings from additional survey questions further accentuated the geographic and financial challenges participants face in accessing specialist care. Participants with access to specialist care travel an average of 414 miles $(S D=790)$ for an appointment. The geographic sparseness of U.S. specialists was apparent in examining the U.S. Census regions of participants with regular specialist care (see Figure 1); no participants in the West North Central or East South Central regions saw specialists as their primary care provider.

Financial barriers to specialist access were revealed through a comparison of the annual household incomes of participants with their use of specialist care. Participants who had never seen a specialist reported significantly lower household incomes $(M=\$ 55,599, \mathrm{SD}=\$ 58,495)$ than those with regular visits to specialist care $(M=\$ 85,284, S D=\$ 116,056)$, Welch's $F(3,199.06)=4.29, p=0.006$. Thus, specialist care is utilized by individuals with higher incomes, yet the onset of ME and CFS can lead to income reductions. Almost half of participants in this study's sample were on disability, and, on average, participants lost $\$ 32,884$ in annual income after becoming ill. Therefore, income reduction represents a significant barrier to specialist care for many individuals with ME and CFS.

Finally, participants were queried about their level of satisfaction with specialist and non-specialist care. Participants who had seen a specialist (regardless of frequency of visits, office visits, or consultations) were asked about their level of agreement with nine statements related to satisfaction with the care they received from their specialist provider. Similarly, all participants (except those who saw a specialist as their primary care provider for their illness) were asked to rate the non-specialist physician that they saw most regularly for their illness using the same nine statements. Table 3 displays the ratings of specialist and non-specialist care; and Table 4 compares the percent of participants who agreed (or strongly agreed) with each statement when referring to specialist care or non-specialist care. While $74.3 \%$ of participants agreed or strongly agreed that they were satisfied with their specialist 
appointments, $45.7 \%$ were satisfied with non-specialist appointments. The majority of participants agreed or strongly agreed that the specialist provided them with helpful information (77.0\%) and helped them manage symptoms $(67.5 \%)$, while fewer agreed or strongly agreed with those statements when asked about non-specialists (20.2\% and $44.1 \%$, respectively). Most participants agreed or strongly agreed that specialists were knowledgeable about the symptoms and course of ME and CFS (91.2\%), treatments (84.4\%), and research findings (86.0\%). In comparison, few participants believed that the non-specialist physicians were knowledgeable about symptoms and course of illness (31.6\%), treatments $(23.3 \%)$, or research findings $(18.2 \%)$.

Table 3. Patient satisfaction with specialist and non-specialist care

\begin{tabular}{|c|c|c|c|c|}
\hline \multirow[b]{3}{*}{ Patient Satisfaction with Specialist Care } & \multicolumn{4}{|c|}{ Percent Agreement } \\
\hline & Strongly & & & Strongly \\
\hline & Disagree & Disagree & Agree & Agree \\
\hline Overall, I feel satisfied with my appointments & 9.0 & 16.7 & 37.0 & 37.3 \\
\hline Provides helpful information on ME or CFS symptoms / treatments & 7.2 & 15.8 & 36.7 & 40.3 \\
\hline Helps me manage my ME or CFS symptoms & 9.2 & 23.4 & 34.4 & 33.1 \\
\hline Knowledgeable about the symptoms/course of ME or CFS & 3.1 & 5.7 & 31.3 & 59.8 \\
\hline Knowledgeable about treatments for ME or CFS & 4.7 & 10.9 & 29.9 & 54.4 \\
\hline Knowledgeable about recent ME or CFS research findings & 5.1 & 8.9 & 32.0 & 54.0 \\
\hline Seems to care about my quality of life & 5.5 & 7.1 & 31.6 & 55.8 \\
\hline Seems to have negative attitudes/beliefs about individuals with ME or CFS & 69.6 & 19.8 & 3.9 & 6.7 \\
\hline Includes me in decisions about my medical care & 4.6 & 8.1 & 32.7 & 54.6 \\
\hline \multicolumn{5}{|l|}{ Patient Satisfaction with Non-Specialist Care } \\
\hline Overall, I feel satisfied with my appointments & 17.7 & 36.5 & 34.4 & 11.4 \\
\hline Provides helpful information on ME or CFS symptoms / treatments & 43.6 & 36.1 & 13.9 & 6.4 \\
\hline Helps me manage my ME or CFS symptoms & 25.2 & 30.7 & 34.7 & 9.4 \\
\hline Knowledgeable about the symptoms/course of ME or CFS & 34.0 & 34.4 & 23.9 & 7.7 \\
\hline Knowledgeable about treatments for ME or CFS & 38.3 & 38.4 & 16.1 & 7.2 \\
\hline Knowledgeable about recent ME or CFS research findings & 44.4 & 37.4 & 11.7 & 6.5 \\
\hline Seems to care about my quality of life & 8.9 & 13.6 & 39.4 & 38.1 \\
\hline Seems to have negative attitudes/beliefs about individuals with ME or CFS & 35.2 & 38.7 & 16.5 & 9.6 \\
\hline Includes me in decisions about my medical care & 6.9 & 10.4 & 46.1 & 36.6 \\
\hline
\end{tabular}

Table 4. Comparison of patient satisfaction with specialist and non-specialist care

\begin{tabular}{lcc}
\hline & \% Agreement or Strong Agreement \\
Satisfaction Domain & Specialist Care & Non-Specialist Care \\
\hline Overall, I feel satisfied with my appointments & 74.3 & 45.7 \\
Provides helpful information on ME or CFS symptoms / treatments & 77.0 & 20.2 \\
Helps me manage my ME or CFS symptoms & 67.5 & 44.1 \\
Knowledgeable about the symptoms/course of ME or CFS & 91.2 & 31.6 \\
Knowledgeable about treatments for ME or CFS & 84.4 & 23.3 \\
Knowledgeable about recent ME or CFS research findings & 86.0 & 18.2 \\
Seems to care about my quality of life & 87.4 & 77.5 \\
Seems to have negative attitudes/beliefs about individuals with ME or CFS & 10.6 & 26.1 \\
Includes me in decisions about my medical care & 87.3 & 82.7
\end{tabular}




\section{Discussion}

The results of this survey clearly indicate that individuals with ME and CFS face significant geographical and financial barriers to accessing specialist care. Furthermore, participants reported substantially less satisfaction with non-specialist care than specialist care. Taken together, these results imply that the majority of individuals with ME and CFS are not receiving what they consider to be a satisfactory level of care and face significant barriers to accessing care. Therefore, these individuals constitute a medically-underserved population.

These results are in sharp contrast to similar studies of individuals with other chronic illnesses. For example, a study of over 2,000 individuals with Multiple Sclerosis (MS) [9], found that $41.3 \%$ saw an MS specialist as their regular care provider. In comparison, just $11.6 \%$ of the current study's sample regularly accessed specialist care. While only $11.6 \%$ of participants with MS reported impediments to accessing healthcare, and none of these participants reported geographic barriers to obtaining care, the results of the current study demonstrate that over $70 \%$ of participants with ME and CFS do not have specialist care in their area. In contrast to the sparse specialist care sites for individuals with ME and CFS, the National MS Society [10] identifies 122 Centers for Comprehensive MS Care across 35 states in the United States. Finally, less than $10 \%$ of participants with MS reported any dissatisfaction with their MS-related healthcare, [9] whereas $25.7 \%$ of participants with ME and CFS reported dissatisfaction with specialist care, and $54.2 \%$ reported dissatisfaction with non-specialist care. While literature suggests that ME and CFS impacts a larger proportion of the population than MS, [11,12] individuals with ME and CFS seem to face disproportionately greater impediments to accessing quality healthcare.

ME and CFS Centers of Excellence could improve access to quality care by reducing the geographic and financial barriers that individuals with ME and CFS currently face. Establishing Centers of Excellence across the United States would reduce the distance between patient and specialist, thereby affording the individual the opportunity to more regularly access specialist care. Furthermore, these centers would provide training opportunities for physicians who are not familiar with the diagnosis and treatment of ME and CFS, satisfying the need and demand for increased numbers of physicians capable of delivering appropriate and specialized care. These additional training and employment opportunities for physicians specialized in ME and CFS could encourage an even larger number of physicians to enter this field by modeling the viability of this particular, specialized medical practice. Although the Centers for Disease Control and Prevention (CDC) has attempted to provide medical education to physicians on ME and CFS (e.g. CDC's Medscape course, "train-the-trainer" models, etc. [13]), continued patient dissatisfaction with medical care suggests that physicians may require longer-term mentorship in the ME and CFS diagnosis and treatment. Such mentorship could be provided at established Centers of Excellence. Moreover, Centers of Excellence, as currently conceived, could engage in local, medical community outreach activities to spread awareness of diagnostic and treatment practices to primary care physicians across the United States. These outreach activities would reduce the disparity between specialist and non-specialist medical care, thereby improving the quality of care for individuals who remain unable to access a Center of Excellence. In addition to reducing geographic barriers to quality care, researchers and clinicians from various Centers of Excellence could engage in interdisciplinary collaborations that would accelerate the pace of research and provide a higher standard of care to an increased number of individuals with ME and CFS.

While the creation of Centers of Excellence requires funding, the economic and societal cost of the status quo greatly exceeds the funding that these centers require. Jason, Benton, Valentine, Johnson, and Torres-Harding [14] found that the direct and indirect economic costs to society incurred due to this illness exceed $\$ 18.7$ billion annually, or $\$ 22,342$ per patient. However, this study applied a prevalence estimate based on the U.S. population in 1999. Applying the prevalence rate (0.42\%) to today's population (318.9 million [15]) indicates annual direct and indirect costs in excess of $\$ 29.9$ billion. These economic costs translate into direct costs to the U.S. government in the form of disability payments, Medicaid benefits, and lost income tax revenue, among other expenses. Despite these substantial costs, CFS and ME remain among the least-funded illnesses by the National Institutes of Health (NIH), with $\$ 5$ million in funding in 2015. [16] NIH funding in 2015 for other chronic illnesses included $\$ 10$ million for fibromyalgia, $\$ 66$ million for Crohn's disease, $\$ 99$ million for Lupus, and $\$ 103$ million for MS. Reducing this spending disparity and improving access to healthcare for individuals with ME and CFS 
would lead to substantial short- and long-term cost savings, as well as increased tax revenues that would surpass the costs associated with establishing and maintaining Centers of Excellence.

The Disability Rights movement demonstrates a precedent for the creation of government-sponsored Centers of Excellence to provide care for individuals who lacked equal access to healthcare, and were, therefore, medically underserved. In 1963, Public Law 88-164 appropriated $\$ 110$ million to establish facilities devoted to research, clinical care, and community outreach for individuals with intellectual disabilities. [17] This law laid the groundwork for the proliferation of centers across the United States. These centers are devoted to serving the needs of individuals with intellectual and developmental disabilities by advancing diagnostics, treatment, and community education. Today, this network of centers is comprised of 67 University Centers for Excellence, 39 Leadership Education in Neurodevelopmental Disabilities programs, and 15 Intellectual and Developmental Disability Research Centers. These programs provide community outreach and education, train health professionals to provide competent, interdisciplinary care, conduct research, and train early-career investigators to promote future research in the field. [18] Public Law 88-164 [17] and the ensuing Developmental Disabilities Assistance and Bill of Rights Act of 2000 [19] establish a precedent and model for the creation of multiple Centers of Excellence for ME and CFS.

The recent Pathways to Prevention report, sponsored by the National Institutes of Health [5], recommended the creation of Centers of Excellence and increased funding for ME and CFS research. The Institute of Medicine [20] echoed this call for increased funding. Given the severity of disability and the decrement in quality of life imparted by ME and CFS symptoms, coupled with the illness's associated economic costs, the recommendations of these two independent reports to create Centers of Excellence for ME and CFS are well supported. Our data suggest that individuals with ME and CFS constitute a medically-underserved population by virtue of geographic and economic barriers. In consequence, individuals with ME and CFS are precluded from accessing appropriate healthcare options and the opportunity to regain reasonable quality of life and self-sufficiency. Equal access to medical care can be achieved by establishing multiple Centers of Excellence, funded at levels commensurate with the number of persons ill with ME and CFS and the degree of illness severity.

Acknowledgments. Funding was provided by the Eunice Kennedy Shriver National Institute of Child Health and Human Development (Grant Number R01HD072208) and the National Institute of Allergy and Infectious Diseases (Grant Number AI105781).

\section{References}

1. T. M. Tidmore, L. A. Jason, L. Chapo-Kroger, S. So, A. Brown, and M. C. Silverman, "Lack of Knowledgeable Healthcare Access for Patients with Neuro-endocrine-immune Diseases," Frontiers in Clinical Medicine, vol. 2, pp. 46-54, 2015.

2. L. A. Jason, E. Paavola, N. Porter, and M. L. Morello, "Frequency and content analysis of chronic fatigue syndrome in medical text books," Australian Journal of Primary Health, vol. 16, no. 2, pp. 174-178, 2010.

3. J. Bowen, D. Pheby, A. Charlett, and C. McNulty, "Chronic Fatigue Syndrome: A survey of GPs' attitudes and knowledge," Family Practice, vol. 22, pp. 389-393, 2005.

4. L. Chapo-Kroger, "Center of Excellence for Myalgic Encephalomyelitis (ME)." [Online]. Available: http://www.hhs.gov/advcomcfs/meetings/presentations/1214-lori-chapo.pdf

5. C. R. Green, P. Cowan, R. Elk, K. M. O'Neil, and A. L. Rasmussen, "National Institutes of Health Pathways to Prevention Workshop: Advancing the Research on Myalgic Encephalomyelitis/Chronic Fatigue Syndrome," Annals of Internal Medicine, vol. 162, pp. 860-865, 2015.

6. K. Friedman, "Myalgic Encephalomyelitis/Chronic Fatigue Syndrome (ME/CFS) Center of Excellence Working Group Report: Justifying ME/CFS Centers of Excellence: The Choices." Presentation to the Chronic Fatigue Syndrome Advisory Committee [Online]. Available: http://www.hhs.gov/advcomcfs/meetings/presentations/day1-combined-deck-dec-2014.pdf, 2015.

7. Chronic Fatigue Syndrome Advisory Committee, "Committee Recommendations." [Online]. Available: https://wayback.archive

it.org/3919/20140324192828/http://www.hhs.gov/advcomcfs/recommendations/09272004.html\#, 2015. 
8. P. A. Harris, R. Taylor, R. Thielke, J. Payne, N. Gonzalez, and J. G. Conde, "Research electronic data capture (REDCap)-A metadata-driven methodology and workflow process for providing translational research informatics support," Journal of Biomedical Informatics, vol. 42, pp. 377-381, 2009.

9. S. L. Minden, D. Frankel, L. Hadden, and D. C. Hoaglin, "Access to health care for people with multiple sclerosis," Multiple Sclerosis, vol. 13, pp. 547-558, 2007.

10.National Multiple Sclerosis Society, "Partners in MS Care." [Online]. Available: http://www.nationalmssociety.org/Treating-MS/Find-an-MS-Care-Provider/Partners-in-MS-Care, 2015.

11.L. A. Jason, J. A. Richman, A. W. Rademaker, K. M. Jordan, A. V. Plioplys, R. R. Taylor, et al., "A community-based study of chronic fatigue syndrome," Archives of Internal Medicine, vol. 159, no. 18, pp. 21292137, 1999.

12.National Multiple Sclerosis Society, "Estimating the prevalence of MS." [Online]. Available: http://www.nationalmssociety.org/About-the-Society/MS-Prevalence, 2015.

13.D. J. Brimmer, K. K. McCleary, T. A. Lupton, K. M. Faryna, K. Hynes, and W. C. Reeves, "A train-the-trainer education and promotion program: Chronic fatigue syndrome-A diagnostic and management challenge," BMC Medical Education, vol. 8, no. 49, doi: 10.1186/1472-6920-8-49, 2008.

14.L. A. Jason, M. C. Benton, L. Valentine, A. Johnson, and S. Torres-Harding, "The Economic impact of ME/CFS: Individual and societal costs," Dynamic Medicine, vol. 7, doi:10.1186/1476-5918-7-6, 2008.

15.United States Census Bureau, "Annual Estimates of the Resident Population: April 1, 2010 to July 1, 2014." [Online data file]. Available:

http://factfinder.census.gov/faces/tableservices/jsf/pages/productview.xhtml?pid=PEP_2014_PEPANNRES\&sr $\mathrm{c}=\mathrm{pt}, 2014$.

16.National Institutes of Health, "Estimates of Funding for Various Research, Condition, and Disease Categories (RCDC)." [Online data file]. Available: http://report.nih.gov/categorical_spending.aspx, 2015.

17. Mental Retardation Facilities and Community Health Centers Construction Act of 1963, Pub. L. 88-164, 77 Stat 282.

18.Association of University Centers on Disabilities. "Network Centers." [Online]. Available: https://www.aucd.org/template/page.cfm?id=2, 2015.

19.Developmental Disabilities Assistance and Bill of Rights Act of 2000, Pub. L. 106-402, 114 Stat. 1677.

20.Institute of Medicine. Beyond myalgic encephalomyelitis/chronic fatigue syndrome: Redefining an illness. Washington, DC: The National Academies Press, 2015. 